\title{
On Coordinated Checkpointing in Distributed Systems
}

\author{
Guohong Cao, Student Member, IEEE, and Mukesh Singhal, Member, IEEE
}

\begin{abstract}
Coordinated checkpointing simplifies failure recovery and eliminates domino effects in case of failures by preserving a consistent global checkpoint on stable storage. However, the approach suffers from high overhead associated with the checkpointing process. Two approaches are used to reduce the overhead: First is to minimize the number of synchronization messages and the number of checkpoints, the other is to make the checkpointing process nonblocking. These two approaches were orthogonal in previous years until the Prakash-Singhal algorithm [18] combined them. In other words, the Prakash-Singhal algorithm forces only a minimum number of processes to take checkpoints and it does not block the underlying computation. However, we found two problems in this algorithm. In this paper, we identify these problems and prove a more general result: There does not exist a nonblocking algorithm that forces only a minimum number of processes to take their checkpoints. Based on this general result, we propose an efficient algorithm that neither forces all processes to take checkpoints nor blocks the underlying computation during checkpointing. Also, we point out future research directions in designing coordinated checkpointing algorithms for distributed computing systems.
\end{abstract}

Index Terms-Distributed system, coordinated checkpointing, causal dependence, nonblocking, consistent checkpoints.

\section{INTRODUCTION}

$\mathrm{C}$ OORDINATED checkpointing is an attractive approach for transparently adding fault tolerance to distributed applications without requiring additional programmer efforts. In this approach, the state of each process in the system is periodically saved on stable storage, which is called a checkpoint of the process. To recover from a failure, the system restarts its execution from a previous error-free, consistent global state recorded by the checkpoints of all processes. More specifically, the failed processes are restarted on any available machine and their address spaces are restored from their latest checkpoints on stable storage. Other processes may have to rollback to their checkpoints on stable storage in order to restore the entire system to a consistent state.

A system state is said to be consistent if it contains no orphan message, i.e., a message whose receive event is recorded in the state of the destination process, but its send event is lost [10], [22]. In order to record a consistent global checkpoint on stable storage, processes must synchronize their checkpointing activities. When a process takes a checkpoint, it asks (by sending checkpoint requests to) all relevant processes to take checkpoints. Therefore, coordinated checkpointing suffers from high overhead associated with the checkpointing process.

Much of the previous work [6], [9], [10], [13] in coordinated checkpointing has focused on minimizing the number of synchronization messages and the number of checkpoints during checkpointing. However, these algorithms

\footnotetext{
- The authors are with the Department of Computer and Information Science, Ohio State University, Columbus, OH 43210.

E-mail: \{gcao, singhal\}@cis.ohio-state.edu.
}

Manuscript received 27 Jan. 1997.

For information on obtaining reprints of this article, please send e-mail to: tpds@computer.org, and reference IEEECS Log Number 100387. (called blocking algorithms) force all relevant processes in the system to block their computations during checkpointing. Checkpointing includes the time to trace the dependence tree and to save the states of processes on stable storage, which may be long. Therefore, blocking algorithms may dramatically degrade system performance [3], [7].

Recently, nonblocking algorithms [7], [20] have received considerable attention. In these algorithms, processes need not block during checkpointing by using a checkpointing sequence number to identify orphan messages. However, these algorithms [7], [20] assume that a distinguished initiator decides when to take a checkpoint. Therefore, they suffer from the disadvantages of centralized algorithms, such as poor reliability, bottleneck, etc. Moreover, these algorithms [7], [20] require all processes in the system to take checkpoints during checkpointing, even though many of them may not be necessary. If they are modified to permit more processes to initiate checkpointing processes, which makes them distributed, these algorithms will suffer from another problem: In order to keep the checkpoint sequence number updated, any time a process takes a checkpoint, it has to notify all processes in the system. If each process can initiate a checkpointing process, the network would be flooded with control messages and processes might waste their time taking unnecessary checkpoints.

Prakash-Singhal algorithm [18] was the first algorithm to combine these two approaches. More specifically, it forces only a minimum number of processes to take checkpoints and does not block the underlying computation during checkpointing. However, we found two problems in this algorithm. In this paper, we identify these two problems and prove a more general result: There does not exist a nonblocking algorithm that forces only a minimum number of processes to take their checkpoints. Based on this general 
result, we propose an efficient nonblocking algorithm which significantly reduces the number of checkpoints. Also, we point out future research directions in designing coordinated checkpointing algorithms for distributed computing systems.

The rest of the paper is organized as follows. Section 2 presents preliminaries. In Section 3, we prove that there does not exist a nonblocking algorithm which forces only a minimum number of processes to take their checkpoints. Section 4 presents the nonblocking checkpointing algorithm. Correctness proofs are provided in Section 5. In Section 6, we compare the proposed algorithm with the existing algorithms. Section 7 presents future research directions and concludes the paper. Problems of the Prakash-Singhal algorithm are described in the Appendix.

\section{Preliminaries}

\subsection{System Model}

The distributed computation we consider consists of $N$ spatially separated sequential processes denoted by $P_{1}, P_{2}, \cdots, P_{N}$. The processes do not share a common memory or a common clock. Message passing is the only way for processes to communicate with each other. The computation is asynchronous, i.e., each process progresses at its own speed and messages are exchanged through reliable channels, whose transmission delays are finite but arbitrary. The messages generated by the underlying distributed application are referred to as computation messages. The messages generated by processes to advance checkpoints are referred to as system messages.

Each checkpoint taken by a process is assigned a unique sequence number. The $i$ th $(i \geq 0)$ checkpoint of process $P_{p}$ is assigned a sequence number $i$ and is denoted by $C_{p, i}$. We also assume that each process $P_{p}$ takes an initial checkpoint $C_{p, 0}$ immediately before execution begins and ends with a virtual checkpoint that represents the last state attained before termination [16]. The ith checkpoint interval of process $P_{p}$ denotes all the computation performed between its $i$ th and $(i+1)$ th checkpoint, including the $i$ th checkpoint but not the $(i+1)$ th checkpoint.

\subsection{Minimizing Dependence Information}

In order to record the dependence relationship among processes, we use a similar approach as the Prakash-Singhal algorithm [18], where each process $P_{i}$ maintains a Boolean vector $R_{i}$, which has $n$ bits. $R_{i}[j]=1$ represents that $P_{i}$ depends on $P_{j}$. At $P_{i}$, the vector is initialized to 0 except $P_{i}[i]$.

When process $P_{i}$ sends a computation message $m$ to $P_{j}$, it appends $R_{i}$ to $m$. After receiving $m, P_{j}$ includes the dependences indicated in $R_{i}$ into its own $R_{j}$ as follows: $R_{j}[k]=R_{j}[k]$ $\vee m \cdot R[k]$, where $1 \leq k \leq n$, and $\vee$ is the bitwise inclusive $O R$ operator. Thus, if a sender $P_{i}$ of a message depends on a process $P_{k}$ before sending the computation message, the receiver $P_{j}$ also depends on $P_{k}$ through transitivity. In Fig. 1, $P_{2}$ depends on $P_{1}$ because of $m 1$. When $P_{3}$ receives $m 2, P_{3}$ (transitively) depends on $P 1$.

The dependence information can be used to reduce the number of processes that must participate in the checkpointing process and the number of messages required to

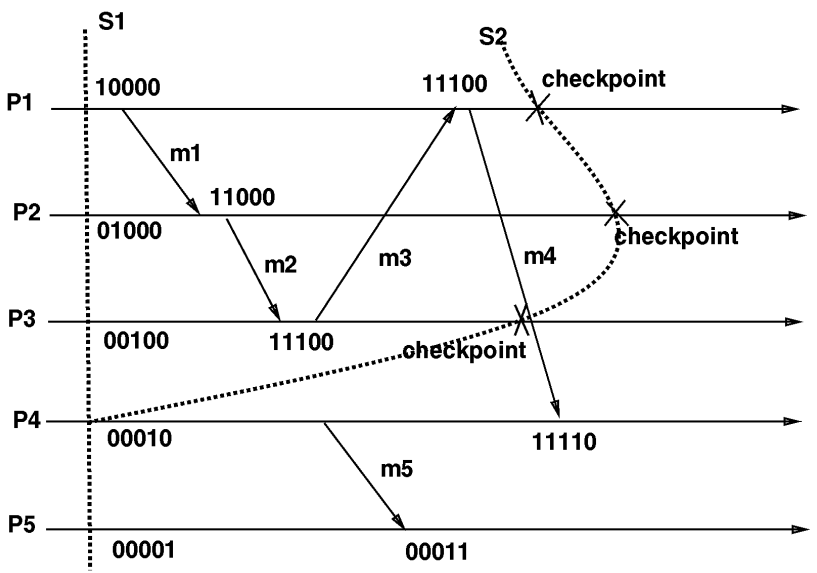

Fig. 1. Checkpointing and dependence information.

synchronize the checkpointing activity. In Fig. 1, the vertical line $S_{1}$ represents the global consistent checkpoints at the beginning of the computation. Later, when $P_{3}$ initiates a new checkpointing process, it only sends checkpoint requests to $P_{1}$ and $P_{2}$. As a result, only $P_{1}, P_{2}$, and $P_{3}$ take new checkpoints. $P_{4}$ and $P_{5}$ continue their computation without taking new checkpoints.

\subsection{Basic Ideas Behind Nonblocking Algorithms}

Most of the existing coordinated checkpointing algorithms [6], [10], [13] rely on the two-phase protocol and save two kinds of checkpoints on stable storage: tentative and permanent. In the first phase, the initiator takes a tentative checkpoint and forces all relevant processes to take tentative checkpoints. Each process informs the initiator whether it succeeded in taking a tentative checkpoint and blocks until released by the initiator in phase two. A process may refuse to take a checkpoint depending on its underlying computation. After the initiator has received positive acknowledgments from all relevant processes, the algorithm enters the second phase. If the initiator learns that all processes have successfully taken tentative checkpoints, it asks them to make their tentative checkpoints permanent; otherwise, it asks them to discard their tentative checkpoints. A process, on receiving the message from the initiator, acts accordingly.

A nonblocking checkpointing algorithm does not require any process to suspend its underlying computation. When processes do not suspend their computations, it is possible for a process to receive a computation message from another process which is already running in a new checkpoint interval. If this situation is not properly dealt with, it may result in an inconsistency. For example, in Fig. 2, $P_{2}$ initiates a checkpointing process. After sending checkpoint requests to $P_{1}$ and $P_{3}, P_{2}$ continues its computation. $P_{1}$ receives the checkpoint request and takes a new checkpoint, then it sends $m 1$ to $P_{3}$. Suppose $P_{3}$ processes $m 1$ before it receives the checkpoint request from $P_{2}$. When $P_{3}$ receives the checkpoint request from $P_{2}$, it takes a checkpoint (see Fig. 2). In this case, $m 1$ becomes an orphan.

Most nonblocking algorithms [7], [20] use a Checkpoint Sequence Number ( $\operatorname{cs} n)$ to avoid inconsistencies. In these algorithms, a process is forced to take a checkpoint if it receives a computation message whose $\operatorname{csn}$ is greater than its 


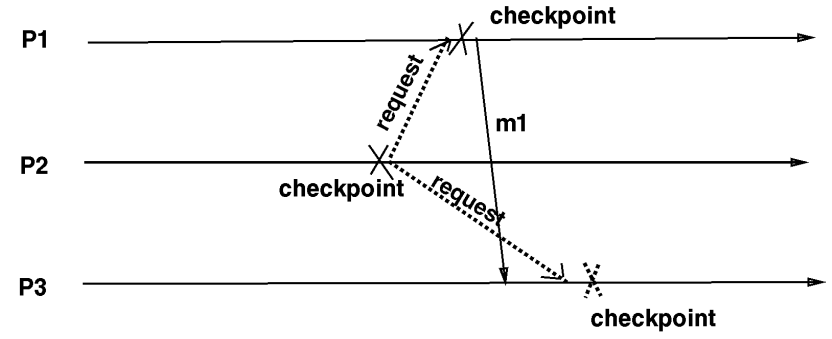

Fig. 2. Inconsistent checkpoints.

local csn. For example, in Fig. 2, $P_{1}$ increases its $\operatorname{csn}$ after it takes a checkpoint and appends the new $\operatorname{csn}$ to $m 1$. When $P_{3}$ receives $m 1$, it takes a checkpoint before processing $m 1$ since the csn appended to $m 1$ is larger than its local $\operatorname{cs} n$.

This scheme works only when every process in the computation can receive each checkpoint request and then increases its own csn. Since the Prakash-Singhal algorithm [18] forces only a subset of processes to take checkpoints, the csn of some processes may be out-of-date and may not be able to avoid inconsistencies. The Prakash-Singhal algorithm attempts to solve this problem by having each process maintain an array to save the $c s n$, where $\operatorname{cs} n_{i}[i]$ is the expected $c s n$ of $P_{i}$. Note that $P_{i}^{\prime} \operatorname{s} \operatorname{cs} n_{i}[i]$ may be different from $P_{j}^{\prime} \operatorname{sis} n_{j}[i]$ if there has been no communication between $P_{i}$ and $P_{j}$ for several checkpoint intervals. By using $\operatorname{cs} n$ and the initiator identification number, they claim that their nonblocking algorithm can avoid inconsistencies and minimize the number of checkpoints during checkpointing. However, we found two problems in their algorithm (see the Appendix). Next, we prove a more general result: "There does not exist a nonblocking algorithm that forces only a minimum number of processes to take their checkpoints."

\section{Proof of IMPossibILITY}

Before presenting the proof, we define a new concept called "z-dependence," which is more general than causal dependence and can be used to model coordinated checkpointing.

Definition 1. If a process $P_{p}$ sends a message to a process $P_{q}$ during its ith checkpoint interval and $P_{q}$ receives the message during its $j$ th checkpoint interval, then $P_{q} \mathbf{z}$-depends on $P_{p}$ during $P_{p}^{\prime}$ s ith checkpoint interval and $P_{q}^{\prime} s j$ th checkpoint interval, denoted as $P_{p} \prec_{j}^{i} P_{q}$.

DEFINITION 2. If $P_{p} \prec_{j}^{i} P_{q}$ and $P_{q} \prec_{k}^{j} P_{r}$, then $P_{r}$ transitively zdepends on $P_{p}$ during $P_{r}^{\prime}$ s $k$ th checkpoint interval and $P_{p}^{\prime} s$ ith checkpoint interval, denoted as $P_{p}{ }^{*}{ }_{k}^{i} P_{r}$ (we simply call it " $P_{r}$ transitively z-depends on $P_{p}$ " if there is no confusion).

PROPOSITION 1.

$$
\begin{aligned}
& P_{p}{ }_{j}^{i} P_{q} \Rightarrow P_{p}{ }^{*}{ }_{j}^{i} P_{q} \\
& P_{p}{ }^{*}{ }_{j}^{i} P_{q} \wedge P_{q}{ }^{*}{ }_{k}^{j} P_{r} \Rightarrow P_{p}{ }^{*}{ }_{k}^{i} P_{r} .
\end{aligned}
$$

The definition of " $z$-dependence" here is different from the concept of "causal dependence" used in the literature.

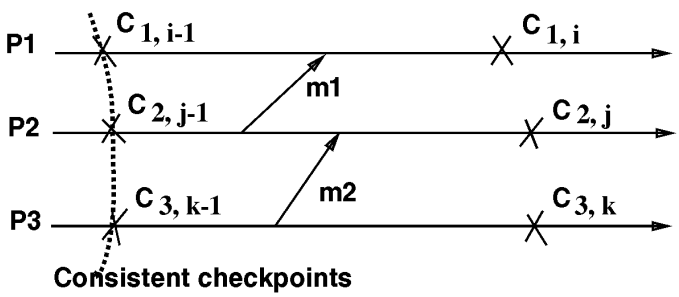

Fig. 3. The difference between causal dependence and z-dependence.

We illustrate the difference between causal dependence and $\mathrm{z}$-dependence in Fig. 3. Since $P_{2}$ sends $m 1$ before it receives $m 2$, there is no causal dependence between $P_{1}$ and $P_{3}$ due to these messages. However, these messages do establish a zdependence between $P_{3}$ and $P_{1}$ :

$$
P_{3}{ }_{j-1}^{k-1} P_{2} \wedge P_{2} \prec_{i-1}^{j-1} P_{1} \Rightarrow P_{3}{ }^{*}{ }_{i-1}^{k-1} P_{1}
$$

DEFINITION 3. A min-process checkpointing algorithm is an algorithm satisfying the following condition: When a process $P_{p}$ initiates a new checkpointing process and takes a checkpoint $C_{p, i}$ a process $P_{q}$ takes a checkpoint $C_{q, j}$ associated with $C_{p, i}$ if and only if $P_{q}{ }^{*}{ }_{i-1}^{j-1} P_{p}$.

In coordinated checkpointing, to avoid an inconsistency, the initiator forces all dependent processes to take checkpoints. For any process, after it takes a checkpoint, it recursively forces all dependent processes to take checkpoints. The Koo-Toueg algorithm [10] uses this scheme, and it has been proven [10] that this algorithm forces only a minimum number of processes to take checkpoints. In the following, we prove that the Koo-Toueg algorithm is a min-process algorithm and a min-process algorithm forces only a minimum number of processes to take checkpoints. To simplify the proof, we use " $P_{p} \vdash{ }_{i}^{j} P_{q}$ " to represent the following: $P_{q}$ causally depends on $P_{p}$ when $P_{q}$ is in the $i$ th checkpoint interval and $P_{p}$ is in the $j$ th checkpoint interval.

PROPOSITION 2.

$$
\begin{aligned}
& P_{p} \vdash{ }_{i}^{j} P_{q} \Rightarrow P_{p} \prec{ }_{i}^{*} P_{q} \\
& P_{p} \prec{ }_{i}^{j} P_{q} \Rightarrow P_{p} \vdash{ }_{i}^{j} P_{q} .
\end{aligned}
$$

LEMMA 1. An algorithm forces only a minimum number of processes to take checkpoints if and only if it is a min-process algorithm.

PROOF. It has been proven [10] that the Koo-Toueg algorithm forces only a minimum number of processes to take checkpoints, thus, we only need to prove the following: In [10], when a process $P_{p}$ initiates a new checkpointing process and takes a checkpoint $C_{p, i}$, a process $P_{q}$ takes a checkpoint $C_{q, j}$ associated with $C_{p, i}$ if and only if $P_{q}{ }^{*}{ }_{i-1}^{j-1} P_{p}$.

NECESSITY. In [10], when a process $P_{p}$ initiates a new checkpoint $C_{p, i}$ it recursively asks all dependent processes 
to take checkpoints. For example, $P_{p}$ asks $P_{k_{m}}$ to take a checkpoint, $P_{k_{m}}$ asks $P_{k_{m-1}}$ to take a checkpoint, and so on. If a process $P_{q}$ takes a checkpoint $C_{q, j}$ associated with $C_{p, i}$, there must be a sequence:

$$
P_{q} \vdash{ }_{s_{k_{1}}}^{j-1} P_{k_{1}} \wedge P_{k_{1}} \vdash{ }_{s_{k_{2}}}^{s_{k_{1}}} P_{k_{2}} \wedge \cdots \wedge P_{k_{m-1}} \vdash{ }_{s_{k_{m}}}^{s_{k_{m-1}}} P_{k_{m}} \wedge P_{k_{m}} \vdash_{i-1}^{s_{k_{m}}} P_{p}
$$$$
(1 \leq m \leq N)
$$

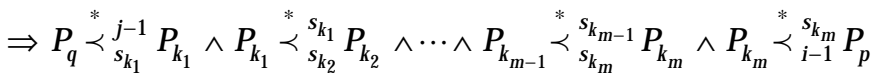

$\Rightarrow P_{q}{ }^{*}{ }_{i-1}^{j-1} P_{p}$

OR

$$
\begin{aligned}
& P_{q} \vdash{ }_{i-1}^{j-1} P_{p} \\
\Rightarrow & P_{q} \prec{ }_{i-1}^{j-1} P_{p} \\
\Rightarrow & P_{q}{ }^{*}{ }_{i-1}^{j-1} P_{p}
\end{aligned}
$$

SUFFICIENCY. If $P_{q} \prec{ }_{i-1}^{j-1} P_{p}$ when $P_{p}$ initiates a new checkpoint $C_{p, i} P_{q}$ takes a checkpoint $C_{q, j}$ associated with $C_{p, i}$ otherwise, if $P_{q}{ }_{i-1}^{*}{ }_{i-1}^{j-1} P_{p}$, there must be a sequence:

$$
\begin{gathered}
P_{q} \prec{ }_{s_{k_{1}}}^{j-1} P_{k_{1}} \wedge P_{k_{1}} \prec{ }_{s_{k_{2}}}^{s_{k_{1}}} P_{k_{2}} \wedge \cdots \wedge P_{k_{m-1}} \prec{ }_{s_{k_{m}}}^{s_{k_{m-1}}} P_{k_{m}} \wedge P_{k_{m}} \prec{ }_{i-1}^{s_{k_{m}}} P_{p} \\
(1 \leq m \leq N) \\
\Rightarrow P_{q} \vdash{ }_{s_{k_{1}}}^{j-1} P_{k_{1}} \wedge P_{k_{1}} \vdash{ }_{s_{k_{2}}}^{s_{k_{1}}} P_{k_{2}} \wedge \cdots \wedge P_{k_{m-1}} \vdash{ }_{s_{k_{m}}}^{s_{k_{m-1}}} P_{k_{m}} \wedge P_{k_{m}} \vdash{ }_{i-1}^{s_{k_{m}}} P_{p}
\end{gathered}
$$

Then, when $P_{p}$ initiates a new checkpoint $C_{p, i}, P_{p}$ asks $P_{k_{m}}$ to take a checkpoint, $P_{k_{m}}$ asks $P_{k_{m-1}}$ to take a checkpoint, and so on. In the end, $P_{k_{1}}$ asks $P_{q}$ to take a checkpoint. Then, $P_{q}$ takes a checkpoint $C_{q, j}$ associated with $C_{p, i}$.

DEFINITION 4. A nonblocking min-process algorithm is a minprocess checkpointing algorithm which does not block the underlying computation during checkpointing.

LEMMA 2. In a nonblocking min-process algorithm, assume $P_{p}$ initiates a new checkpointing process and takes a checkpoint $C_{p, i}$. If a process $P_{r}$ sends a message $m$ to $P_{q}$ after it takes a new checkpoint associated with $C_{p, i}$ then $P_{q}$ takes a checkpoint $C_{q, j}$ before processing $m$ if and only if $P_{q}{ }^{*}{ }_{i-1}^{j-1} P_{p}$.

Proof. From the definition of min-process, $P_{q}$ takes a checkpoint $C_{q, j}$ if and only if $P_{q}{ }^{*}{ }_{i-1}^{j-1} P_{p}$. Thus, we only need to show that $P_{q}$ takes $C_{q, j}$ before processing $m$. It is easy to see, if $P_{q}$ takes $C_{q, j}$ after processing $m, m$ becomes an orphan (as in Fig. 2).

From Lemma 2, in a nonblocking min-process algorithm, when a process receives a message $m$, it must know if the initiator of a new checkpointing process transitively zdepends on it.

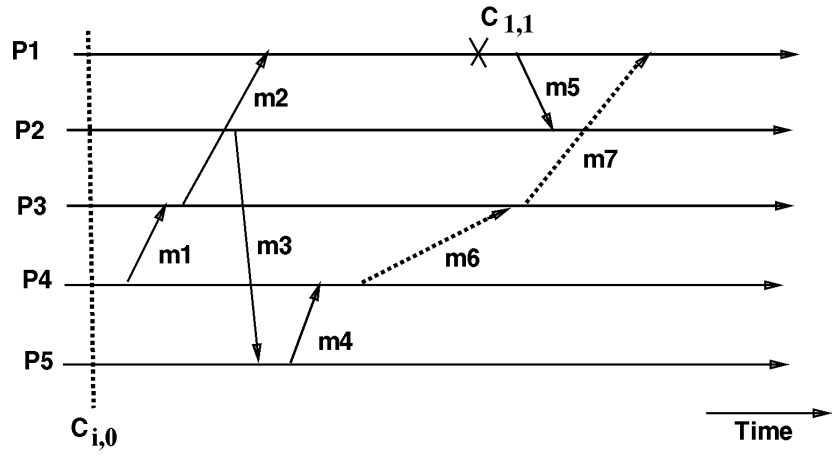

Fig. 4. Tracing the dependence.

LEMMA 3. In a nonblocking min-process algorithm, there is not enough information at the receiver of a message to decide whether the initiator of a new checkpointing process transitively z-depends on the receiver.

PROOF. The proof is by construction (using a counterexample). In Fig. 4, assume messages $m 6$ and $m 7$ do not exist. $P_{1}$ initiates a checkpointing process. When $P_{4}$ receives $m 4$, there is a $\mathrm{z}$-dependence as follows:

$$
P_{2}{ }^{*}{ }_{0}^{0} P_{4} \wedge P_{4}{ }^{*}{ }_{0}^{0} P_{1} \Rightarrow P_{2}{ }^{*}{ }_{0}^{0} P_{1} \text {. }
$$

However, $P_{2}$ does not know this when it receives $m 5$. There are two possible approaches for $P_{2}$ to get the $\mathrm{z}$ dependence information:

APPROACH 1 (Tracing the incoming messages). In this approach, $P_{2}$ gets the new $z$-dependence information from $P_{1}$. Then, $P_{1}$ has to know the $\mathrm{z}$-dependence information before it sends $m 5$ and appends the $\mathrm{z}$ dependence information to $m 5$. In Fig. $4, P_{1}$ cannot get the new z-dependence information $\left(P_{2}{ }_{\prec}^{*}{ }_{0}^{0} P_{1}\right)$ unless $P_{4}$ notifies $P_{1}$ of the new z-dependence information when $P_{4}$ receives $m 4$. There are two ways for $P_{4}$ to notify $P_{1}$ of the new z-dependence information: First is to broadcast the z-dependence information (not illustrated in the figure); the other is to send the $\mathrm{z}$ dependence information by an extra message $m 6$ to $P_{3}$, which in turn notifies $P_{1}$ by $m 7$. Both of them dramatically increase message overhead. Since the algorithm does not block the underlying computation, it is possible that $P_{1}$ receives $m 7$ after it sends out $m 5$ (as shown in the figure). Thus, $P_{2}$ still cannot get the $\mathrm{z}$ dependence information when it receives $m 5$.

APPROACH 2 (Tracing the outgoing messages). In this approach, since $P_{2}$ sends message $m 3$ to $P_{5}, P_{2}$ hopes to get the new $z$-dependence information from $P_{5}$. Then, $P_{5}$ has to know the new $\mathrm{z}$-dependence information and it would like to send an extra message (not shown in the figure) to notify $P_{2}$. Similarly, $P_{5}$ needs to get the new zdependence information from $P_{4}$, which comes from $P_{3}$, and finally from $P_{1}$. Certainly, this requires much more 
extra messages than Approach 1. Similar to Approach 1, $P_{2}$ still cannot get the $z$-dependence information in time since the computation is in progress.

THEOREM 1. No nonblocking min-process algorithm exists.

PROOF. From Lemma 2, in a nonblocking min-process algorithm, a receiver has to know if the initiator of a new checkpointing process transitively z-depends on the receiver, which is impossible from Lemma 3. Therefore, no nonblocking min-process algorithm exists.

COROLLARY 1 . There does not exist a nonblocking algorithm that forces only a minimum number of processes to take their checkpoints.

PROOF. The proof directly follows from Lemma 1 and Theorem 1.

\subsection{Remarks}

Netzer and Xu [16] introduced the concept of "zigzag" paths to define the necessary and sufficient conditions for a set of local checkpoints to lie on a consistent global checkpoint. Our definition of " $z$-dependence" captures the essence of zigzag paths. If an initiator forces all its transitively z-dependent processes to take checkpoints, the resulting checkpoints are consistent, and no zigzag path exists among them. If the resulting checkpoints are consistent, there is no zigzag path among them, and all processes on which the initiator transitively zdepends have taken checkpoints. However, there is a distinct difference between a zigzag path and z-dependence. A zigzag path is used to evaluate whether the existing checkpoints are consistent; thus, it is mainly used to find a consistent global checkpoint in an uncoordinated checkpointing algorithm. It has almost no use in a coordinated checkpointing algorithm, since a consistent global checkpoint is guaranteed by the synchronization messages. The $\mathrm{z}$-dependence is proposed for $\mathrm{CO}^{-}$ ordinated checkpointing and it reflects the whole synchronization process of coordinated checkpointing, e.g., in the proof of Lemma 1, z-dependence is used to model the checkpointing process. Based on $\mathrm{z}$-dependence, we found and proved the impossibility result. It is impossible to prove the result only based on zigzag paths.

Wang [23], [24] considered the problem of constructing the maximum and the minimum consistent global checkpoints that contain a target set of checkpoints. In his work, a graph called rollback dependence graph (or "R-graph") can be used to quickly find zigzag paths. Based on R-graph, Wang developed efficient algorithms to calculate the maximum and the minimum consistent global checkpoints for both general nondeterministic executions and piecewise deterministic executions. Manivannan et al. [14] generalized this problem. They proposed an algorithm to enumerate all such consistent global checkpoints that contain a target set of checkpoints and showed how the minimum and the maximum checkpoints are special cases. Their work [14] is based on a concept called "Z-cone," which is a generalization of zigzag paths. All these works [14], [23], [24] are based on zigzag paths. Similar to [16], these works [14], [23], [24] are not useful in coordinated checkpointing since checkpoints are guaranteed to be consistent in coordinated checkpointing. Thus, the notions of zig-zag paths

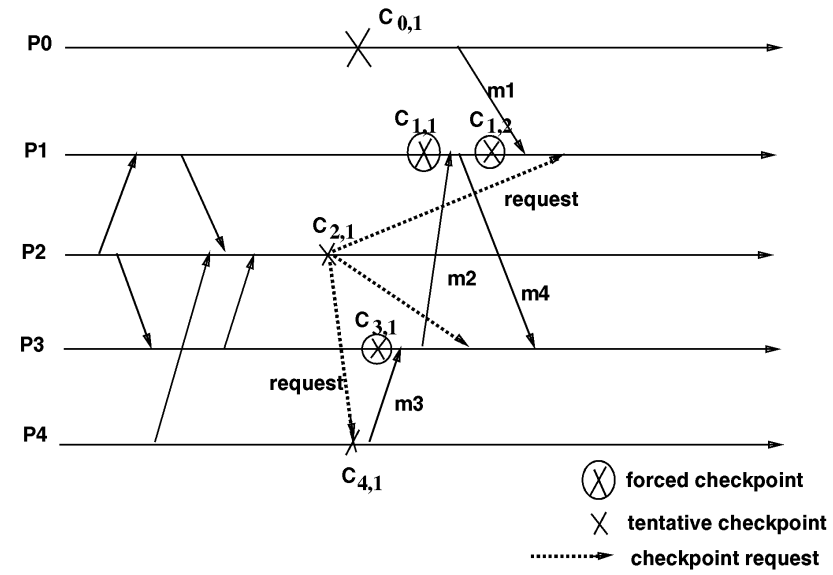

Fig. 5. An example of checkpointing.

and Z-cone help find consistent global checkpoints in uncoordinated checkpointing, while the notion of $z-$ dependence helps understand the nature of coordinated checkpointing.

\section{A CheCKPointing Algorithm}

In this section, we present our checkpointing algorithm, which neither blocks the underlying computation nor forces all processes to take checkpoints.

\subsection{Basic Idea}

\subsubsection{Basic Scheme}

A simple nonblocking scheme for checkpointing is as follows. When a process $P_{i}$ sends a computation message, it piggybacks the current value of $\operatorname{csn}_{i}[i]$ ( $\operatorname{cs} n$ is explained in Section 2.3). When a process $P_{j}$ receives a computation message $m$ from $P_{i}, P_{j}$ processes the message if $m . c s n \leq \operatorname{csn}_{j}[i]$; otherwise, $P_{j}$ takes a checkpoint (called forced checkpoint), updates its $\operatorname{csn}\left(\operatorname{cs} n_{j}[i]=m . \operatorname{csn}\right)$, and then processes the message. This method may result in a large number of checkpoints. Moreover, it may lead to an avalanche effect, in which processes in the system recursively ask others to take checkpoints.

For example, in Fig. 5, to initiate a checkpointing process, $P_{2}$ takes its own checkpoint and sends checkpoint request messages to $P_{1}, P_{3}$, and $P_{4}$. When $P_{2}$ 's request reaches $P_{4}, P_{4}$ takes a checkpoint. Then, $P_{4}$ sends message $m 3$ to $P_{3}$. When $m 3$ arrives at $P_{3}, P_{3}$ takes a checkpoint before processing it due to $m 3 . c s n>\operatorname{csn}_{3}[4]$. For the same reason, $P_{1}$ takes a checkpoint before processing $m 2$.

$P_{0}$ has not communicated with other processes before it takes a local checkpoint. Later, it sends a computation message $m 1$ to $P_{1} . P_{1}$ takes checkpoint $C_{1,2}$ before processing $m 1$, because $P_{0}$ has taken a checkpoint with checkpoint sequence number larger than $P_{1}$ expected. Then, $P_{1}$ requires $P_{3}$ to take another checkpoint (not shown in the figure) due to $m 2$ and $P_{3}$ in turn asks $P_{4}$ to take another checkpoint (not shown in the figure) due to $m 3$. If $P_{4}$ had received messages from other processes after it sent $m 3$, those processes would have been forced to take checkpoints. This chain may never end.

We reduce the number of checkpoints based on the following observation. In Fig. 5, if $m 4$ does not exist, it is not 
necessary for $P_{1}$ to take $C_{1,2}$ since checkpoint $C_{1,1}$ is consistent with the rest of the checkpoints. Based on this observation, we get the following revised scheme.

\subsubsection{Revised Basic Scheme}

"When a process $P_{j}$ receives a computation message $m$ from $P_{i}, P_{j}$ only takes a checkpoint when $m . \operatorname{csn}>\operatorname{csn}_{j}[i]$ and $P_{j}$ has sent at least a message in the current checkpoint interval."

In Fig. 5, if $m 4$ does not exist, $C_{1,2}$ is not necessary according to the revised scheme. However, if $m 4$ exists, the revised scheme still results in a large number of checkpoints and may result in an avalanche effect.

\subsubsection{Enhanced Scheme}

We now present the basic idea of our enhanced scheme that eliminates avalanche effects during checkpointing. From Fig. 5, we make two observations:

OBSERVATION 1. It is not necessary to take checkpoint $C_{1,2}$ even though $m 4$ exists, since $P_{0}$ does not transitively z-depend on $P_{1}$ during the zeroth checkpoint interval of $P_{0}$.

OBSERVATION 2. From Lemma $3, P_{1}$ does not know if $P_{0}$ transitively z-depends on $P_{1}$ when $P_{1}$ receives $m 1$.

These observations imply that $C_{1,2}$ is unnecessary but unavoidable. Thus, there are two kinds of forced checkpoints in response to computation messages. In Fig. 5, $C_{1,1}$ is different from $C_{1,2}$. $C_{1,1}$ is a checkpoint associated with the initiator $P_{2}$, and $P_{1}$ will receive the checkpoint request from $P_{2}$ in the future. $C_{1,2}$ is a checkpoint associated with the initiator $P_{0}$, but $P_{1}$ will not receive the checkpoint request from $P_{0}$ in the future. To avoid an inconsistency, $P_{1}$ should keep $C_{1,1}$ when $P_{1}$ receives $P_{2}$ 's request. However, $P_{1}$ can discard $C_{1,2}$ after a period (defined as the longest checkpoint request travel time). If only one checkpointing process is in progress, $P_{1}$ can also discard $C_{1,2}$ when it needs to make its tentative checkpoint permanent. Thus, our algorithm has three kinds of checkpoints: tentative, permanent, and forced. Tentative and permanent checkpoints are the same as before and they are saved on stable storage. Forced checkpoints do not need to be saved on stable storage. They can be saved anywhere, even in the main memory. When a process takes a tentative checkpoint, it forces all dependent processes to take checkpoints. However, a process taking a forced checkpoint does not require its dependent processes to take checkpoints. Suppose a process $P_{i}$ takes a forced checkpoint associated with an initiator $P_{j}$. When $P_{i}$ receives the corresponding checkpoint request associated with $P_{j}, P_{i}$ converts the forced checkpoint into a tentative checkpoint. After that, $P_{i}$ forces all processes on which it depends to take checkpoints. If $P_{i}$ does not receive the checkpoint request associated with $P_{j}$ after a period or $P_{i}$ needs to make its tentative checkpoint permanent, it may discard the forced checkpoint associated with $P_{j}$ to save storage. Actually, $P_{i}$ may have not finished taking its checkpointing process after a period defined as before; if so, $P_{i}$ stops the ongoing checkpointing and resumes its computation.

In Fig. $5, C_{1,2}$ is a forced checkpoint that will be discarded in the future. Since $C_{1,2}$ is a forced checkpoint, it is not necessary for $P_{3}$ to take a new checkpoint when $P_{1}$ takes $C_{1,2}$ as in the previous scheme. Thus, our scheme avoids the avalanche effect and improves the performance of checkpointing.

\subsection{Data Structures}

The following data structures are used in our algorithm:

$R_{i}$ : A Boolean vector described in Section 2.2. The operator $\ominus$ is defined as follows:

$$
i \ominus j= \begin{cases}1 & \text { if } i=1 \wedge j=0 \\ 0 & \text { otherwise }\end{cases}
$$

$\operatorname{cs} n_{i}$ : An array of $n$ checkpoint sequence numbers ( $\left.\operatorname{csn} n\right)$ at each process $P_{i}$. Each checkpoint sequence number is represented by an integer. $c s n_{i}[j]$ represents the checkpoint sequence number of $P_{j}$ that $P_{i}$ knows. In other words, $P_{i}$ expects to receive a message from $P_{j}$ with checkpoint sequence number $\operatorname{csn}_{i}[j]$. Note that $\operatorname{csn}_{i}[i]$ is the checkpoint sequence number of $P_{i}$.

weight: A nonnegative variable of type real with maximum value of 1 . It is used to detect the termination of the checkpointing algorithm as in [8].

first $_{i}$ : A Boolean array of size $n$ maintained by each process $P_{i}$. The array is initialized to all zeroes each time a checkpoint at that process is taken. When $P_{i}$ sends a computation message to process $P_{j}, P_{i}$ sets first $\left.i j\right]$ to one.

trigger $_{i}$ : A tuple (pid, inum) maintained by each process $P_{i}$. pid indicates the checkpointing initiator that triggered this process to take its latest checkpoint. inum indicates the csn at process pid when it took its own local checkpoint on initiating the checkpointing process. trigger is appended to each system message and the first computation message to which a process sends after taking its local checkpoint.

$C P_{i}$ : A list of records maintained by each process $P_{i}$. Each record has the following fields:

forced: forced checkpoint information of $P_{i}$.

$R: P_{i}^{\prime}$ s own Boolean vector before it takes the current forced checkpoint, but after it takes the previous forced checkpoint or permanent checkpoint, whichever is later.

trigger: a set of triggers. Each trigger represents a different initiator. $P_{i}$ may have one common forced checkpoint for several initiators.

first: $P_{i}$ 's own first before it takes the current forced checkpoint, but after it takes the previous forced checkpoint or permanent checkpoint, whichever is later.

The following notations are used to access these records:

$C P[i][k]$ is the $k$ th record of $P_{i}$;

$C P[i][$ last $]$ is the last record of $P_{i}$;

$C P[i][$ last +1$]$ will be a new record appended at the end of $P_{i}^{\prime}$ s records.

csn is initialized to an array of zeros at all processes. The trigger tuple at process $P_{i}$ is initialized to $(i, 0)$. The weight at a process is initialized to zero. When a process $P_{i}$ sends any computation message, it appends its $\operatorname{CSn}_{i}[i]$ and $R_{i}$ to 
the message. Also, $P_{i}$ appends its trigger in the first computation message it sends to every other process after taking its checkpoint. $P_{i}$ checks if $s e n d_{i}[j]=0$ before sending a computation message to $P_{j}$. If so, it sets $s e n d_{i}[j]$ to one and appends its trigger to the message.

\subsection{The Checkpointing Algorithm}

To clearly present the algorithm, we assume that at any time, at most one checkpointing process is in progress. Techniques to handle concurrent initiations of checkpointing by multiple processes can be found in [17]. As multiple concurrent initiations of checkpointing are orthogonal to our discussion, we only briefly mention the main features of [17]. When a process receives the first request for checkpointing initiated by another process, it takes a local checkpoint and propagates the request. All local checkpoints taken by the participating processes for a checkpoint initiation collectively form a global checkpoint. The state information collected by each independent checkpointing is combined. The combination is driven by the fact that the union of consistent global checkpoints is also a consistent global checkpoint. The checkpoints thus generated are more recent than each of the checkpoints collected independently and also more recent than that collected by [21]. Therefore, the amount of computation lost during rollback, after process failures, is minimized. Next, we present our nonblocking algorithm.

Checkpointing initiation. Any process can initiate a checkpointing process. When a process $P_{i}$ initiates a checkpointing process, it takes a local checkpoint, increments its $\operatorname{csn}_{i}[i]$, sets weight to one, and stores its own identifier and the new $\operatorname{csn}_{i}[i]$ in its trigger. Then, it sends a checkpoint request to each process $P_{j}$ such that $R_{i}[j]=1$ and resumes its computation. Each request carries the trigger of the initiator, the $R_{i}$, and a portion of the weight of the initiator, whose weight is decreased by an equal amount.

Reception of checkpoint requests. When a process $P_{i}$ receives a request from $P_{j}$, it compares $P_{j}$.trigger (msg_trigger) with $P_{i}$.trigger (own_trigger). If these two triggers are different, $P_{i}$ checks if it has sent any message during the current checkpoint interval. If $P_{i}$ has not sent a message during the current checkpoint interval, it updates its csn, sends a reply to the initiator with the weight equal to the weight received in the request, and resumes its underlying computation; otherwise, $P_{i}$ takes a tentative checkpoint and propagates the request as follows. For each process $P_{k}$ on which $P_{i}$ depends but $P_{j}$ does not $\left(P_{j}\right.$ has sent request to the processes on which it depends), $P_{i}$ sends a request to $P_{k}$. Also, $P_{i}$ appends the initiator's trigger and a portion of the received weight to all those requests. Then, $P_{i}$ sends a reply to the initiator with the remaining weight and resumes its underlying computation.

If msg_trigger is equal to own_trigger when $P_{i}$ receives a request (implying that $P_{i}$ has already taken a checkpoint for this checkpointing process), $P_{i}$ does not need to take a checkpoint. But,

- If there is a forced checkpoint whose trigger is equal to $m s g \_$trigger, $P_{i}$ saves the forced checkpoint on stable storage (the forced checkpoint is turned into a tentative checkpoint). Then, $P_{i}$ propagates the request as before.

- If there is not a forced checkpoint whose trigger is equal to msg_trigger, a reply is sent to the initiator with the weight equal to that received in the request.

Computation messages received during checkpointing. When $P_{i}$ receives a computation message $m$ from $P_{j}, P_{i}$ compares m.csn with its local $\operatorname{cs} n_{i}[j]$. If $m . \operatorname{csn} \leq \operatorname{cs} n_{i}[j]$, the message is processed and no checkpoint is taken; otherwise, it implies that $P_{j}$ has taken a checkpoint before sending $m$ and $m$ is the first computation message sent by $P_{j}$ to $P_{i}$ after $P_{j}$ took its checkpoint. Therefore, the message $m$ must have a trigger tuple. $P_{i}$ first updates its $\operatorname{csn}_{i}[j]$ to $m . c s n$, then does the following depending on the information of $P_{j}$.trigger (msg_trigger) and $P_{i}$.trigger (own_trigger):

- If msg_trigger $=$ own_trigger, it means that the latest checkpoints of $P_{i}$ and $P_{j}$ were both taken in response to the same checkpoint initiation. Therefore, no new local checkpoint is needed.

- If msg_trigger $\neq$ own_trigger, $P_{i}$ checks if it has sent any message during the current checkpoint interval. If $P_{i}$ has not sent any message during the current checkpoint interval, it only needs to add msg_trigger to its forced checkpoint list. If $P_{i}$ has sent a message during the current checkpoint interval, it takes a forced checkpoint and updates its data structures such as csn, $C P, R$, and first.

Termination and garbage collection. The checkpoint initiator adds weights received in all reply messages to its own weight. When its weight becomes equal to one, it concludes that all processes involved in the checkpointing process have taken their tentative local checkpoints. As a result, it sends out commit messages to all processes from which it received replys. Processes make their tentative checkpoints permanent on receiving the commit messages. The older permanent local checkpoints and all forced checkpoints at these processes are discarded because a process will never roll back to a point prior to the newly committed checkpoint. Note that when a process discards its forced checkpoints, it also updates its data structures such as $C P, R$, and first.

A formal description of the checkpointing algorithm is given below:

type trigger $=$ record (pid, inum: integer;) end

var own_trigger, msg_trigger: trigger;

csn: array[1..n] of integers;

weight: real;

$C P$ : array[1..n] of LIST;

process_set: set of integers;

$R$, temp1, temp2, first: bit array of size $n$;

Actions taken when $P_{i}$ sends a computation message to $P_{j}$ : if first $_{i}[j]=0$

then first $_{i}[j]:=1$; $\operatorname{send}\left(P_{i}\right.$, message, $R_{i}, \operatorname{csn}_{i}[i]$, own_trigger $)$; else send $\left(P_{i}\right.$, message, $R_{i}, \operatorname{csn}_{i}[i]$, NULL);

Actions for the initiator $P_{j}$ :

take a local checkpoint (on stable storage);

$\operatorname{increment}\left(\operatorname{csn}_{j}[j]\right)$; own_trigger $:=\left(P_{j}, \operatorname{csn}_{j}[j]\right)$; clear process_set;

prop_cp $\left(R_{j}, \mathrm{NULL}, P_{j}\right.$, own_trigger, 1.0$)$; 
Other processes, $P_{i}$, on receiving a checkpoint request from $P_{j}$ :

receive $\left(P_{j}\right.$, request, $m . R$, recv_csn, msg_trigger, recv_weight); $\operatorname{csn}_{j}[j]:=$ recv_csn;

if msg_trigger $=$ own_trigger

then if $\exists k$ (msg_trigger $\in C P[i][k])$

then save $C P[i][k]$.forced on stable storage; $\operatorname{prop} \_c p\left(R_{i}, m . R, P_{i}, \mathrm{msg}\right.$ _trigger, recv_weight);

else send $\left(P_{i}\right.$, reply, recv_weight) to the initiator;

else if first $_{i}[]=0$

then send $\left(P_{i}\right.$, reply, recv_weight) to the initiator;

else take a local checkpoint (on stable storage); increment $\left(\operatorname{csn}_{i}[i]\right)$; own_trigger := msg_trigger; prop_cp $\left(R_{i}, m . R, P_{i}\right.$, msg_trigger, recv_weight);

Actions for process $P_{i}$, on receiving a computation message from $P_{j}$ :

receive $\left(P_{j}, \mathrm{~m}, \mathrm{~m} . R\right.$, recv_csn, msg_trigger);

if recv_csn $\leq c s n_{i}[j]$

then process the message and exit;

else $c s n_{i}[j]:=$ recv_csn;

if msg_trigger $=$ own_trigger

then process the message;

else if first $_{i}[]=0$

then if $C P[i] \neq \phi$ then

$C P[i][$ last $]$.trigger $:=C P[i][$ last $]$.trigger $\cup$ msg_trigger

else take a checkpoint, save it in $C P[i][$ last +1$]$.forced;

$C P[i][$ last +1$]$.trigger $:=$ msg_trigger;

$C P[i][$ last +1$] . R:=R_{i}$; reset $R_{i}$ and first $t_{i}$

own_trigger $:=$ msg_trigger; increment $\left(\operatorname{cs} n_{i}[i]\right)$;

process the message;

$\operatorname{prop} \_c p\left(R_{i}, m . R, P_{i}\right.$, msg_trigger, recv_weight)

if $\exists k$ (msg_trigger $\in C P[i][k]$.trigger $)$

then temp $1:=C P[i][k] . R$;

for $\mathrm{j}:=0$ to $\mathrm{k}-1$ do temp $1:=$ temp $1 \cup C P[i][j] . R$

else temp1 $:=R_{i}$;

for $\mathrm{j}:=0$ to last do temp $1:=$ temp $1 \cup C P[i][j] . R$

temp $2:=$ temp $1 \ominus m . R$; temp $1:=$ temp1 OR $m . R$;

weight := recv_weight;

for any processes $P_{k}$, such that temp2[k] = 1

weight $:=$ weight $/ 2$;

send $\left(P_{i}\right.$, request, temp $1, \operatorname{csn}_{i}[i]$, msg_trigger, weight); send $\left(P_{i}\right.$, reply, weight) to the initiator; reset $R_{i}$ and first $t_{i}$;

Actions in the second phase for the initiator $P_{i}$ :

Receive $\left(P_{j}\right.$, reply, recv_weight)

weight $:=$ weight + recv_weight;

process_set := process_set $\cup P_{j}$;

if weight $=1$

then for any $P_{k}$, such that $P_{k} \in$ process_set,

send(commit, msg_trigger) to $P_{k}$;

Actions for other process $P_{j}$ :

if Receive(commit, msg_trigger)

then make the tentative checkpoint permanent;

find the $k$, such that (msg_trigger $\in C P[i][k]$.trigger);

for $\mathrm{i}:=\mathrm{k}+1$ to last do $R_{j}:=R_{j} \cup C P[j][i] . R$;

for $\mathrm{i}:=\mathrm{k}+1$ to last do first $_{j}:=$ first $_{j} \cup C P[j][i]$.first;

clear the forced checkpoint list.

\subsection{An Example}

The basic idea of the algorithm can be better understood by the example presented in Fig. 5. To initiate a checkpoint, $P_{2}$ takes its own checkpoint and sends checkpoint request messages to $P_{1}, P_{3}$ and $P_{4}$, since $R_{2}[1]=1, R_{2}[3]=1$, and $R_{2}[4]=1$. When $P_{2}$ 's request reaches $P_{4}, P_{4}$ takes a checkpoint. Then, $P_{4}$ sends a computation message $m 3$ to $P_{3}$. When $m 3$ arrives at $P_{3}, P_{3}$ takes a forced checkpoint before processing it because $m 3 . c s n>\operatorname{csn}_{3}[4]$ and $P_{3}$ has sent a message during the current checkpoint interval. For the same reason, $P_{1}$ takes a forced checkpoint before processing $m 2$.

$P_{0}$ has not communicated with other processes before it takes a local checkpoint. Later, it sends a computation message $m 1$ to $P_{1} . P_{1}$ takes a checkpoint $C_{1,2}$ before processing $m 1$, because $P_{0}$ has taken a checkpoint with checkpoint sequence number larger than $P_{1}$ expected and $P_{1}$ has sent $m 4$ during the current checkpoint interval.

When $P_{1}$ receives the request from $P_{2}$, it searches its forced checkpoint list. Since $C_{1,1}$ is a forced checkpoint associated with $P_{2}, P_{2}$ turns $C_{1,1}$ into a tentative checkpoint by saving it on stable storage. Similarly, $P_{3}$ converts $C_{3,1}$ to a tentative checkpoint when it receives the checkpoint request from $P_{2}$. Finally, the checkpointing process initiated by $P_{2}$ terminates when checkpoints $C_{1,1}, C_{2,1}, C_{3,1}$, and $C_{4,1}$ are made permanent. $P_{1}$ discards $C_{1,2}$ when it makes checkpoint $C_{1,1}$ permanent.

\subsection{Discussion}

Checkpointing includes the time to trace the dependence tree and to save the states of processes on stable storage, which may be long. For a network of powerful workstations connected by a high-speed network, even a small blocking time may result in substantial lost computation, but taking an extra checkpoint does not consume much time on a powerful workstation and this time may be much less than the blocking time. Moreover, based on the empirical study of [3], [7], blocking the underlying computation may dramatically reduce the system performance. Thus, our nonblocking algorithm is much more efficient than blocking algorithms for a network of workstations.

Coordinated checkpointing algorithms suffer from the synchronization overhead associated with the checkpointing process. During the last decade, the communication overhead far exceeded the overhead of accessing stable storage [3]. Furthermore, the memory available to run processes tended to be small. These trade-offs naturally favored uncoordinated checkpointing schemes over coordinated checkpointing schemes. In modern systems, the overhead of coordinating checkpoints is negligible compared to the overhead of saving the states [7], [15]. Using concurrent and incremental checkpointing [7], the overhead of either coordinated or uncoordinated checkpointing is essentially the same. Therefore, uncoordinated checkpointing is not likely to be an attractive technique in practice given the negligible performance gains. These gains do not justify the complexities of finding a consistent recovery line after the failure, the susceptibility to the domino effect, the high stable storage overhead of saving multiple checkpoints of each process, and the overhead of garbage collection. Thus, our coordinated 
checkpointing algorithm has many advantages over uncoordinated checkpointing algorithms.

Due to technological advancements, the synchronization cost is negligible compared to the overhead of saving the states on stable storage [7], [15]. Stable storage for checkpoints is provided by a highly available network file server. Checkpoints cannot be saved on a local disk or in local volatile or nonvolatile memory since that will make them inaccessible during an extended outage of the local machine. The cost of saving checkpoints on stable storage therefore includes both the cost of network transmission to the file server and the cost of accessing the stable storage device on the file server. Since most of the processes take checkpoints almost at the same time, the stable storage at the file server is more likely to be a bottleneck. Our algorithm requires minimum number of processes to take tentative checkpoints and thus minimizes the workload at the stable storage server. The "forced checkpoints" do not need to be saved on stable storage. They can be saved anywhere, even in the local volatile memory. Thus, taking a "forced checkpoint" avoids the cost of transferring large amounts of data to the stable storage and accessing the stable storage device, and thus it has much less overhead compared to taking a tentative checkpoint on stable storage. Also, by taking "forced checkpoints," our algorithm avoids the avalanche effect and significantly reduces the number of checkpoints.

In our algorithm, the number of "forced checkpoints" depends on the application. A process takes a "forced checkpoint" only when it receives a computation message which has a csn larger than the process expects. Further reducing the number of "forced checkpoints" will be our future work. Overall, our algorithm is efficient in the sense that it is nonblocking, requires minimum stable storage, minimizes the number of tentative checkpoints, and avoids the avalanche effect.

\section{Correctness Proof}

In Section 2.2, $R_{i}$ represents all dependence relations in the current checkpoint period. Due to forced checkpoints, $R_{i}$ represents all dependence relations after the last forced checkpoint or tentative checkpoint, whichever is later. Note that a process saves its current $R_{i}$ and then clears it whenever it takes a forced checkpoint. As a result, we use Boolean array temp 1 to recalculate the dependence relations in the current checkpoint interval, which is a sequence of union operations depending on the number of forced checkpoints taken. To simplify the proof, we use $R_{i}$ to represent all dependence relations in the current checkpoint interval. If there are forced checkpoints in the current checkpoint period, $R_{i}$ represents the value of temp 1 .

LEMMA 4. If process $P_{i}$ takes a checkpoint and $R_{i}[j]=1, P_{j}$ takes a checkpoint for the same checkpointing initiation.

ProOF. If $P_{i}$ is an initiator, it sends checkpoint request messages to all processes such that $R_{i}[j]=1$. If $P_{i}$ is not an initiator, it takes a checkpoint on receiving a request from a process $P_{k}$. For each process $P_{j}$ such that $R_{i}[j]=1$, there are two possibilities:
CASE 1. If $\mathrm{m} \cdot R[\mathrm{j}]=0$ in the request received by $P_{i}$ from $P_{k}, P_{i}$ sends a request to $P_{j}$.

CASE 2. If $\mathrm{m} \cdot R[\mathrm{j}]=1$ in the request received by $P_{i}$ from $P_{k}$, a request has been sent to $P_{j}$ by at least one process in the checkpoint request propagation path from the initiator to $P_{k}$.

Therefore, if a process takes a checkpoint, every process on which it depends receives at least one checkpoint request. There are three possibilities when $P_{j}$ receives the first checkpoint request:

1) $P_{j}$ has taken a tentative checkpoint for this checkpoint initiation when the first checkpoint request arrives: This request and all subsequent request messages for this initiation are ignored.

2) $P_{j}$ has not taken a tentative checkpoint or a forced checkpoint for this checkpoint initiation when the first request arrives: $P_{j}$ takes a checkpoint on receiving the request.

3) $P_{j}$ has taken a forced checkpoint for this checkpoint initiation when the first checkpoint request arrives: $P_{j}$ turns the forced checkpoint into a tentative checkpoint and all subsequent request messages for this initiation are ignored.

Hence, when a process takes a checkpoint, each process on which it depends also takes a checkpoint. These dependencies may have been present before the checkpointing process was initiated, or may have been created while the coordinated checkpointing process was in progress.

THEOREM 2. The algorithm creates a consistent global checkpoint.

PrOOF. We prove this by contradiction. Assume that the global state of the system is inconsistent at a time instance. Then, there must be a pair of processes $P_{i}$ and $P_{j}$ such that at least one computation message $m$ has been sent from $P_{j}$ after $P_{j}^{\prime}$ s last checkpoint and has been received by $P_{i}$ before $P_{i}$ 's last checkpoint. So, $R_{i}[j]=1$ at $P_{i}$ at the time of taking its checkpoint. From Lemma 4, $P_{j}$ has taken a checkpoint. There are three possibilities under which $P_{j}^{\prime}$ s checkpoint is taken:

CASE 1. $P_{j}^{\prime}$ s checkpoint is taken due to a request from $P_{i}$. Then:

$\operatorname{send}(m)$ at $P_{j} \rightarrow{ }^{1}$ receive $(\mathrm{m})$ at $P_{i}$ receive $(\mathrm{m})$ at $P_{i} \rightarrow$ checkpoint taken at $P_{i}$ checkpoint taken at $P_{i} \rightarrow$ request sent by $P_{i}$ to $P_{j}$ request sent by $P_{i}$ to $P_{j} \rightarrow$ checkpoint taken at $P_{j}$

Using the transitivity property of $\rightarrow$, we have: send $(\mathrm{m})$ at $P_{j} \rightarrow$ checkpoint taken at $P_{j}$. Thus, the sending of $m$ is recorded at $P_{j}$.

CASE 2. $P_{j}$ 's last checkpoint is taken due to a request from a process $P_{k}, k \neq i$. According to the assumption, $P_{j}$ sends $m$ after taking its local checkpoint which is triggered by $P_{k}$. When $m$ arrives at $P_{i}$, there are two possibilities:

CASE 2.1. $P_{i}$ has taken a forced checkpoint for this checkpoint initiation. Then, $P_{i}$ processes $m$ as 
normal. When $P_{i}$ receives a checkpoint request for this checkpoint initiation, $P_{i}$ turns the forced checkpoint into a tentative checkpoint. As a result, the reception of $m$ is not recorded in the checkpoint of $P_{i}$.

CASE 2.2. $P_{i}$ has not taken a forced checkpoint for this checkpoint initiation. Since $\operatorname{csn}$ appended with $m$ is greater than $P_{i} \cdot \operatorname{cs} n[j], P_{i}$ takes a forced checkpoint before processing $m$. When $P_{i}$ receives a checkpoint request for this checkpoint initiation, $P_{i}$ turns the forced checkpoint into a tentative checkpoint. Certainly, the reception of $m$ is not recorded in the checkpoint of $P_{i}$.

CASE 3. $P_{j}$ 's checkpoint is taken due to the arrival of a computation message $m^{\prime}$ at $P_{j}$ from $P_{k}$. Similar to Case 2, we have a contradiction.

THEOREM 3. The checkpointing algorithm terminates within a finite time.

PROOF. The proof is similar to [18].

\section{Related Work}

The first coordinated checkpointing algorithm was presented in [2]. However, it assumes that all communications between processes are atomic, which is too restrictive. The Koo-Toueg algorithm [10] relaxes this assumption. In this algorithm, only those processes that have communicated with the checkpoint initiator either directly or indirectly since the last checkpoint need to take new checkpoints. Thus, it reduces the number of synchronization messages and the number of checkpoints. Later, Leu and Bhargava [13] presented an algorithm which is resilient to multiple process failures. Also, this algorithm does not assume that the channel is FIFO, which is necessary in [10]. However, these two algorithms [10], [13] assume a complex scheme (such as slide window) to deal with the message loss problem and do not consider lost messages in checkpointing and recovery. Deng and Park [6] proposed an algorithm to address both orphan message and lost messages.

In Koo and Toueg's algorithm [10], if any of the involved processes is not able to or not willing to take a checkpoint, the entire checkpointing process is aborted. Kim and Park [9] proposed an improved scheme that allows the new checkpoints in some subtrees to be committed while the others are aborted.

To further reduce the system messages needed to synchronize the checkpointing, loosely synchronous clocks [5], [19] are used. More specifically, loosely synchronized checkpoint clocks can trigger the local checkpointing actions of all participating processes at approximately the same time without the need of broadcasting the checkpoint request by an initiator. However, a process taking a checkpoint needs to wait for a period that equals the sum of the maximum deviation between clocks and the maximum time to detect a failure in another process in the system.

All the above coordinated checkpointing algorithms [2], [5], [6], [9], [10], [13], [19] require processes to be blocked during checkpointing. Checkpointing includes the time to trace the dependence tree and to save the state of processes on stable storage, which may be long. Therefore, blocking algorithms may dramatically reduce system performance [3], [7].

The Chandy-Lamport algorithm [4] is the earliest nonblocking algorithm for coordinated checkpointing. However, in their algorithm, system messages (markers) are sent along all channels in the network during checkpointing. This leads to a message complexity of $O\left(n^{2}\right)$. Moreover, it requires all processes to take checkpoints and the channel must be FIFO. To relax the FIFO assumption, Lai and Yang [11] proposed another algorithm. In their algorithm, when a process takes a checkpoint, it piggybacks a checkpoint request (a flag) to the messages it sends out from each channel. The receiver checks the piggybacked message flag to see if there is a need to take a checkpoint before processing the message. If so, it takes a checkpoint before processing the message to avoid an inconsistency. To record the channel information, each process needs to maintain the entire message history on each channel as part of the local checkpoint. Thus, the space requirements of the algorithm may be large. Moreover, it requires all processes to take checkpoints, even though many of them are unnecessary.

In [25], when a process takes a checkpoint, it may continue its normal operation without blocking, because processes keep track of any delayed message. This algorithm is based on the idea of atomic send-receive checkpoints. Each sender and receiver make the balance between the messages exchanged, and keep the set of unbalanced messages as part of checkpoint data. However, this scheme requires each process to log each message sent, which may introduce some performance degradation and require the system to be deterministic.

The Elnozahy-Johnson-Zwaenepoel algorithm [7] uses a checkpoint sequence number to identify orphan messages, thus avoiding the need for processes to be blocked during checkpointing. However, this approach requires all processes to take checkpoints during checkpointing. The algorithm proposed by Silva and Silva [20] uses a similar idea as [7] except that the processes which did not communicate with others during the previous checkpoint interval do not need to take new checkpoints. Both algorithms [7], [20] assume that a distinguished initiator decides when to take a checkpoint. Therefore, they suffer from the disadvantages of centralized algorithms such as one-site failure, traffic bottle-neck, etc. Moreover, their algorithms require almost all processes to take checkpoints, even though many of them are unnecessary. If they are modified to permit more processes to initiate checkpointing processes, which makes them distributed, the new algorithm suffers from another problem; in order to keep the checkpoint sequence number updated, any time a process takes a checkpoint, it has to notify all processes in the system. If each process can initiate a checkpointing process, the network would be flooded with control messages and processes might waste their time taking unnecessary checkpoints.

All the above algorithms follow two approaches to reduce the overhead associated with coordinated checkpointing 
algorithms: One is to minimize the number of synchronization messages and the number of checkpoints [2], [5], [6], [9], [10], [13], [19], the other is to make checkpointing nonblocking [4], [7], [11], [20]. These two approaches were orthogonal in previous years until the Prakash-Singhal algorithm [18] combined them. However, their algorithm has some problems and may result in inconsistencies. Therefore, our algorithm is the first correct algorithm to combine these two approaches. In other words, our nonlocking algorithm avoids the avalanche effect and significantly reduces the number of checkpoints.

\section{Conclusions}

Coordinated checkpointing has received considerable attention because of its low stable storage requirement and low rollback recovery overhead. However, it suffers from high overhead associated with the checkpointing process. A large number of algorithms have been proposed to reduce the overhead associated with coordinated checkpointing. Compared to these works, this paper has made three contributions to coordinated checkpointing:

1) Based on a new concept "z-dependence," we proved a more general result: There does not exist a nonblocking algorithm that forces only a minimum number of processes to take their checkpoints.

2) Different from the literature, a "forced checkpoint" in our algorithm is neither a tentative checkpoint nor a permanent checkpoint, but it can be turned into a tentative checkpoint. Based on this innovative idea, our nonblocking algorithm avoids the avalanche effect and significantly reduces the number of checkpoints.

3) We identified two problems in the Prakash-Singhal algorithm [18].

From Theorem 1, no nonblocking min-process algorithm exists. This implies that there are three directions in designing efficient coordinated checkpointing algorithms. On one extreme, we proposed a nonblocking algorithm, which relaxed the min-process condition while minimizing the number of tentative checkpoints; future work along this direction consists of further reducing the number of "forced checkpoints." The other extreme is to relax the nonblocking condition while keeping the min-process property, that is, we can design a min-process algorithm which tries to minimize the blocking time. Between these two extremes, we can also design blocking non-min-process algorithms that significantly reduce the blocking time as well as the number of checkpoints. Future research in designing coordinated checkpointing algorithms will consider these three approaches.

Since different applications may have different constraints, identifying suitable applications for these three approaches is valuable. For example, in mobile computing systems, due to vulnerability of Mobile Hosts ( $\mathrm{MHs}$ ) to catastrophic failures, e.g., loss, theft, or physical damage, the disk storage on an $M H$ cannot be considered stable storage. A reasonable solution [1] is to utilize the stable storages at Mobile Support Stations (MSSs) to store checkpoints of $M H$ s. Thus, to take a checkpoint, an

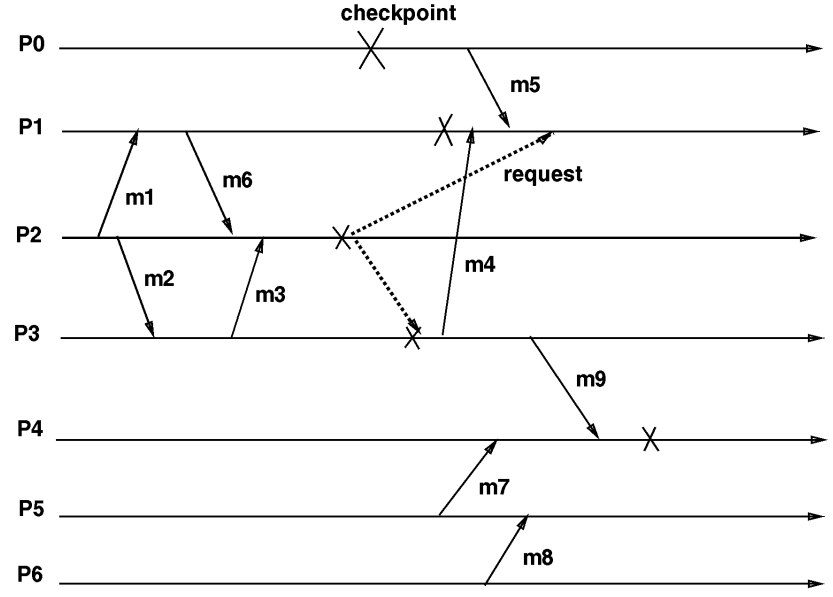

Fig. 6. The first problem.

$M H$ has to transfer a large amount of data to its local MSS over the wireless network. Since the wireless network has low bandwidth, the min-process property is much more important in such environments. Most $\mathrm{MHs}$ have low computation power, a small amount of blocking time does not mean too much lost computation for them, but taking and transferring unnecessary checkpoints may waste a large amount of computation power, bandwidth, and energy. Moreover, the checkpointing may last much longer than the blocking time. Therefore, the min-process property may be more important than the nonblocking property in designing coordinated checkpointing algorithms for mobile computing systems. However, for a network of powerful workstations connected by a highspeed network, even a small blocking time may result in substantial lost computation, but taking an extra checkpoint does not consume much time on a powerful workstation and this time may be much less than the blocking time. Also, since there are no strict bandwidth and energy consumption requirements, the nonblocking property may be more important than the min-process property in designing coordinated checkpointing algorithms for a network of workstations.

\section{APPENDIX}

The coordinated checkpointing algorithm proposed by Prakash and Singhal [18] may result in inconsistencies. In the following, we identify two problems in this algorithm and discuss some possible solutions.

\section{The First Problem}

Prakash and Singhal [18] claim that their algorithm can take lazy checkpoints. In Section 4.4 of their paper, they gave the following example: As in Fig. 6, $P_{3}$ sends $m 9$ to $P_{4}$ after $P_{3}$ finishes taking its checkpoint. When $P_{4}$ receives $m 9$, it takes a new checkpoint (lazy checkpoint). Then, $P_{4}$ asks $P_{5}$ to take a checkpoint and $P_{5}$ asks $P_{6}$ to take a checkpoint. $P_{1}, P_{2}$, or $P_{3}$ do not send checkpoint request to $P_{4}, P_{5}$, and $P_{6}$, since $P_{1}$, $P_{2}$, and $P_{3}$ do not depend on $P_{4}, P_{5}$, or $P_{6}$. The checkpointing algorithm terminates when the weight of $P_{2}$ is equal to one. Because the computation message $m 9$ does not carry any 


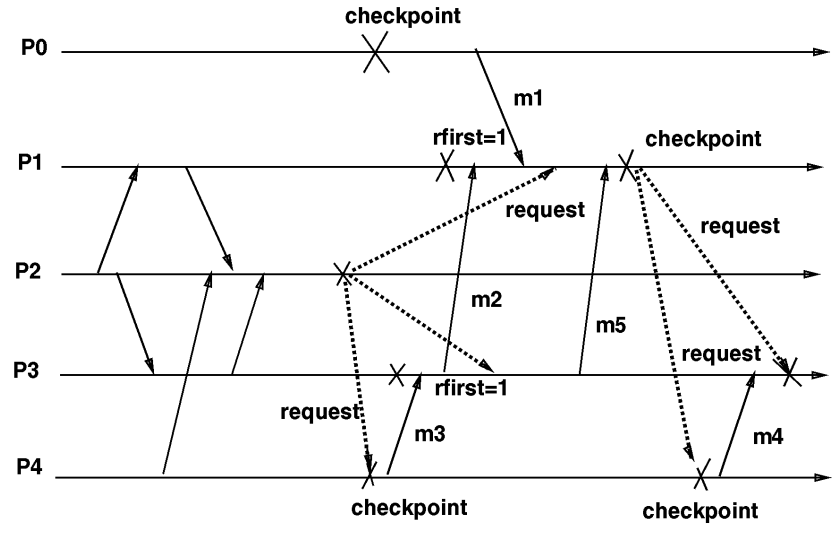

Fig. 7. The second problem.

weight, $P_{4}, P_{5}$, and $P_{6}$ send responses to the initiator with weight 0 . Therefore, the termination of the algorithm is independent of their responses. Suppose $P_{2}$ finds out that its weight becomes equal to one before $P_{6}$ takes its checkpoint, but after $P_{2}$ has received the responses from $P_{4}$ and $P_{5}$. Then, $P_{2}$ sends commit messages to $P_{4}$ and $P_{5}$, but not $P_{6}$. As a result, $P_{4}$ and $P_{5}$ make their tentative checkpoints permanent. If $P_{6}$ fails before it finishes taking its local checkpoint, it results in an inconsistency.

\section{A Solution to the First Problem}

As presented in our algorithm, we solve this problem by using a forced checkpoint. The process taking a forced checkpoint does not propagate the checkpoint request until it receives the checkpoint request associated with this checkpoint initiation. In this example, $P_{4}$ only takes a forced checkpoint, it does not require $P_{5}$ and $P_{6}$ to take checkpoints. The forced checkpoint will be discarded since no process makes it permanent. Note that our algorithm only needs to propagate the checkpoint request once, but the Prakash-Singhal algorithm propagates the checkpoint request twice. However, lazy checkpointing is eliminated. Certainly, a process can always propagate lazy checkpoints by initiating another round of checkpointing. For example, after $P_{4}$ takes a lazy checkpoint, it initiates (as an initiator) another checkpointing process and makes them permanent after $P_{5}$ and $P_{6}$ have taken their checkpoints.

\section{The Second Problem}

We illustrate the second scenario using the example in Fig. 7. $P_{2}$ initiates a checkpointing process by taking its own checkpoint and sends checkpoint request messages to $P_{1}, P_{3}$, and $P_{4}$. When $P_{2}$ 's request reaches $P_{4}, P_{4}$ takes a checkpoint and sends message $m 3$ to $P_{3}$. When $m 3$ arrives at $P_{3}, P_{3}$ takes a checkpoint before processing the message, because $m 3$ is the first message received by $P_{3}$ such that msg_trigger.pid $\neq$ own_trigger.pid. $P_{3}$ sets $r$ first to 1 . For the same reason, $P_{1}$ takes a checkpoint and sets rfirst to 1 before processing $m 2$.

$P_{0}$ has not communicated with other processes before it takes a local checkpoint. Later, it sends a message $m 1$ to $P_{1}$. Because $P_{0}$ has taken a checkpoint, its checkpoint sequence number is larger than $P_{1}$ expected. However, $m 1$ is not the first computation message received by $P_{1}$ with a larger checkpoint sequence number than expected (rfirst $=1$ ). Therefore, no checkpoint is taken.

Suppose $P_{1}$ initiates a checkpointing process after it receives $m 5 . P_{1}$ takes a checkpoint and then it sends checkpoint request messages to $P_{2}, P_{3}$, and $P_{4}$. When $P_{1}$ 's request reaches $P_{4}, P_{4}$ takes a checkpoint. Suppose $P_{4}$ sends a message $m 4$ to $P_{3}$ after it finishes its checkpoint. Because $m 4$ is not the first computation message received by $P_{3}$ with a larger checkpoint sequence number than expected (rfirst is already 1), $P_{3}$ does not take a checkpoint when it receives $m 4$. Later, when $P_{3}$ receives the checkpoint request from $P_{1}$, it takes a checkpoint. As a result, $m 4$ becomes an orphan.

\section{Possible Solutions to the Second Problem}

The problem arises due to the variable rfirst. In Fig. 7, one possible solution is to let $P_{3}$ clear rfirst before it receives $m 4$. In this case, $P_{3}$ will take a checkpoint before processing $m 4$. However, things are not that simple. In order to avoid the avalanche effect or unnecessary checkpoints, there is a need to keep the value of rfirst as long as possible. On the other hand, to avoid an inconsistency, there is a need to clear rfirst as soon as possible. Thus, we have a contradiction.

Another possible solution can be illustrated by Fig. 7 . When $P_{4}$ sends $m 4$, it appends the dependence information to $m 4$. When $P_{3}$ receives $m 4$, it knows that the initiator transitively depends on it, so $P_{3}$ takes a checkpoint before processing $m 4$. However, we can easily construct a counterexample, such as that in Lemma 3. Therefore, to solve the second problem, we have to introduce extra checkpoints.

\section{REFERENCES}

[1] A. Acharya and B.R. Badrinath, "Checkpointing Distributed Applications on Mobil Computers," Proc. Third Int'l Conf. Parallel and Distributed Information Systems, Sept. 1994.

[2] G. Barigazzi and L. Strigini, "Application-Transparent Setting of Recovery Points," Digest of Papers, Proc. 13th Fault Tolerant Computing Symp. (FTCS-13), pp. 48-55, 1983.

[3] B. Bhargava, S.R. Lian, and P.J. Leu, "Experimental Evaluation of Concurrent Checkpointing and Rollback-Recovery Algorithms," Proc. Int'l Conf. Data Eng., pp. 182-189, 1990.

[4] K.M. Chandy and L. Lamport, "Distributed Snapshots: Determining Global States of Distributed Systems," ACM Trans. Computer Systems, Feb. 1985.

[5] F. Cristian and F. Jahanian, "A Timestamp-Based Checkpointing Protocol for Long-Lived Distributed Computations," Proc. IEEE Symp. Reliable Distributed Systems, pp. 12-20, 1991.

[6] Y. Deng and E.K. Park, "Checkpointing and Rollback-Recovery Algorithms in Distributed Systems," J. Systems and Software, pp. 5971, Apr. 1994.

[7] E.N. Elnozahy, D.B. Johnson, and W. Zwaenepoel, "The Performance of Consistent Checkpointing," Proc. 11th Symp. Reliable Distributed Systems, pp. 86-95, Oct. 1992.

[8] S.T. Huang, "Detecting Termination of Distributed Computations by External Agents," Proc. Ninth Int'l Conf. Distributed Computing Systems, pp. 79-84, 1989.

[9] J.L. Kim and T. Park, "An Efficient Protocol For Checkpointing Recovery in Distributed Systems," IEEE Trans. Parallel and Distributed Systems, vol. 5, no. 8, pp. 955-960, Aug. 1993.

[10] R. Koo and S. Toueg, "Checkpointing and Rollback-Recovery for Distributed Systems," IEEE Trans. Software Eng., vol. 13, no. 1, pp. 23-31, Jan. 1987.

[11] T.H. Lai and T.H. Yang, "On Distributed Snapshots," Information Processing Letters, pp. 153-158, May 1987.

[12] L. Lamport, "Time, Clocks and Ordering of Events in Distributed Systems," Comm. ACM, July 1978. 
[13] P.Y. Leu and B. Bhargava, "Concurrent Robust Checkpointing and Recovery in Distributed Systems," Proc. Fourth IEEE Int'l Conf. Data Eng., pp. 154-163, 1988.

[14] D. Manivannan, R. Netzer, and Mukesh Singhal, "Finding Consistent Global Checkpoints in a Distributed Computation," IEEE Trans. Parallel and Distributed Systems, vol. 8, no. 6, pp. 623-627, June 1997.

[15] G. Muller, M. Hue, and N. Peyrouz, "Performance of Consistent Checkpointing in a Modular Operating System: Results of the FTM Experiment," Lecture Notes in Computer Science: Proc. First European Conf. Dependable Computing (EDCC-1), pp. 491-508, Oct. 1994.

[16] R.H.B. Netzer and J. Xu, "Necessary and Sufficient Conditions for Consistent Global Snapshots," IEEE Trans. Parallel and Distributed System, vol. 6, no. 2, pp. 165-169, Feb. 1995.

[17] R. Prakash and M. Singhal, "Maximal Global Snapshot with Concurrent Initiators," Proc. Sixth IEEE Symp. Parallel and Distributed Processing, pp. 344-351, Oct. 1994.

[18] R. Prakash and M. Singhal, "Low-Cost Checkpointing and Failure Recovery in Mobile Computing Systems," IEEE Trans. Parallel and Distributed System, vol. 7, no. 10, pp. 1,035-1,048, Oct. 1996.

[19] P. Ramanathan and K.G. Shin, "Use of Common Time Base for Checkpointing and Rollback Recovery in a Distributed System," IEEE Trans. Software Eng., vol. 19, no. 6, pp. 571-583, June 1993.

[20] L.M. Silva and J.G. Silva, "Global Checkpointing for Distributed Programs," Proc. 11th Symp. Reliable Distributed Systems, pp. 155162, Oct. 1992.

[21] M. Spezialetti and P. Kearns, "Efficient Distributed Snapshots," Proc. Sixth Int'l Conf. Distributed Computing Systems, pp. 382-388, 1986.

[22] R.E. Strom and S.A. Yemini, "Optimistic Recovery in Distributed Systems," ACM Trans. Computer Systems, pp. 204-226, Aug. 1985.

[23] Y. Wang, "Maximum and Minimum Consistent Global Checkpoints and Their Application," Proc. 14th IEEE Symp. Reliable Distributed Systems, pp. 86-95, Oct. 1995.

[24] Y. Wang, "Consistent Global Checkpoints that Contain a Given Set of Local Checkpoints," IEEE Trans. Computers, vol. 46, no. 4, pp. 456-468, Apr. 1997.

[25] Z. Wojcik and B.E. Wojcik, "Fault Tolerant Distributed Computing Using Atomic Send Receive Checkpoints," Proc. Second IEEE Symp. Parallel and Distributed Processing, pp. 215-222, 1990.

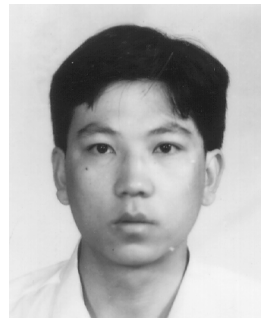

Guohong Cao received his BS degree from Xian Jiaotong University, Xian, China. He received an MS degree in computer science from Ohio State University in 1997 . He is currently a $\mathrm{PhD}$ candidate in the Department of Computer and Information Science at Ohio State University. His research interests include distributed computing systems, fault tolerance, mobile computing, and wireless networks.

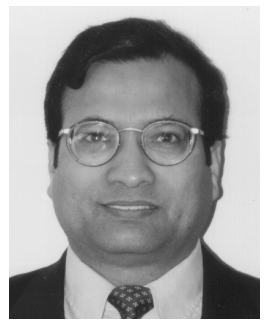

Mukesh Singhal received a Bachelor of Engineering degree in electronics and communication engineering with high distinction from the University of Roorkee, Roorkee, India, in 1980, and a PhD degree in computer science from the University of Marland, College Park, in 1986. He is an associate professor of computer and information science at Ohio State University, Columbus. His current research interests include operating systems, distributed systems, mobile computing, high-speed networks, computer security, and performance modeling. He has published more than 100 refereed articles in these areas. He has coauthored two books: Advanced Concepts in Operation Systems, (McGraw-Hill, 1994) and Readings in Distributed Computing Systems, (IEEE CS Press, 1993). He has served on the program committees of several international conferences and symposiums. He was the program co-chair of the Sixth International Conference on Computer Communications and Networks in 1997 (IC3N '97) and is the program chair of the 17th IEEE Symposium on Reliable Distributed Systems, 1998. 